\title{
Large monolithic silica-based macrocellular foams with trimodal pore system $\dagger$
}

\author{
Lenin Huerta $¥$, Carmen Guillem, Julio Latorre, Aurelio Beltrán, Daniel Beltrán and Pedro Amorós* \\ Institut de Ciència dels Materials (ICMUV), Universitat de València, P. O. Box 2085, 46071-València, \\ Spain.E-mail: pedro.amoros@uv.es; Fax: 3496 3543633; Tel: 34963543617
}

Received (in Cambridge, UK) 12th February 2003, Accepted 25th April 2003

First published as an Advance Article on the web 19th May 2003

Silica-based materials with hierarchical pore systems at three different length scales (small mesopores-large mesopores-macropores) have been prepared through a nanotectonic approach by using mesoporous nanoparticles as building blocks; the resulting materials present a highly accessible foam-like architecture and can be prepared as large monoliths.

The design of new materials with frameworks involving hierarchical pore systems and complex macroscale forms is an emerging area owing to potential applications ${ }^{1}$ on the basis of the possibility of harmonizing an enhanced accessibility to the functional active groups (across large pores) with the conservation of the high surface area and pore structure. ${ }^{2-4}$ These very open networks favour diffusion of reagents and products while avoiding undesired pore blocking phenomena. A variety of bimodal materials combining micro-meso, meso-macro or micro-macroporosity has been described to date. ${ }^{4}$ However, as far as we know, there is only one report dealing with a solid including a trimodal pore system. ${ }^{5}$ The described synthesis approaches are usually based on the use of so many different template agents as pore types. On the other hand, shaping of porous solids in the form of monoliths instead of fine powders (as usually produced) confers on them additional advantages and wide-ranging applications in catalysis. ${ }^{5-8}$ Different research groups have been able to prepare zeolite monoliths with additional macroporous voids by using double scale template agents (small molecular templates and bacterial structures, polystyrene latex spheres or polyurethane foams as secondary large templates), and starting from solutions of inorganic precursors or, alternatively, from preformed zeolite nanoparticles as building blocks. ${ }^{5,9,10}$

Polymer foams have been extensively used to synthesize ceramic foams of several compositions. ${ }^{11}$ These ceramic materials are usually prepared by coating the surface of the polymer with a slurry of ceramic powder; later polymer removal by calcination leads to a ceramic replica of the organic foam. Lee et al. ${ }^{8}$ have recently reported on the use for the first time of polyurethane foams as large-scale secondary templates to prepare micro-macroporous silicalite-1 monoliths using softchemistry conditions typical for obtaining zeolites. In this case, the silicalite crystals grow over the polyurethane surface from a solution confined in the macrocellular voids. In contrast, MCM41 and related mesoporous materials do not present the ability of zeolites to generate large monoliths and multimodal pore systems. Difficulties increase when nanotectonic approaches are intended, taking into account the unavailability of mesoporous nanoparticles on a large scale. Thus, there is no report to date dealing with the preparation of large monoliths using preformed silica mesoporous nanoparticles as building blocks.

Here, we present the preparation and characterization of silica-based large monoliths having trimodal pore systems (small meso-, large meso- and macroporous). The monoliths are

$\dagger$ Electronic supplementary information (ESI) available: $\mathrm{N}_{2}$ adsorptiondesorption isotherm. See http://www.rsc.org/suppdata/cc/b3/b301620j/ $\ddagger$ Departamento de Química, Facultad de Ciencias, Universidad del Zulia, P. O. Box 526, Maracaibo, Venezuela. synthesized through a nanotectonic way by using preformed UVM-7 mesoporous nanoparticles and polyurethane foams as macroscale template. UVM-7 like materials show very open architectures consisting of micrometric aggregates of mesoporous nanoparticles connected through covalent bonds. This organization defines two pore systems: the first one is generated by effect of the surfactant micelles, and the second one is formed in the course of the condensation reactions among the mesoporous nanoparticles. ${ }^{12}$

A simple four-steps scheme of a typical synthesis is as follows: (1) preparation of the mesoporous material; (2) particle-size reduction (from micro to submicro or nanoparticles) by ultrasound irradiation; (3) coating of the polyurethane foam with nanoparticles leading to the foam mineralization, and (4) elimination of the organic foam by calcination. All samples were analyzed and characterized by XRD techniques (Seifert 3000TT using $\mathrm{CuK} \alpha$ radiation), SEM (Philips XL 30ESEM and Hitachi S-4100 FE), TEM (Philips CM10), ${ }^{29} \mathrm{Si}$ MAS NMR (Varian Unity-300), and $\mathrm{N}_{2}$ adsorptiondesorption isotherms (Micromeritics ASAP2010).

The first step is the UVM-7 synthesis. The general procedure, a modification of the so-called atrane route, has been recently described in detail. ${ }^{12}$ The only difference here with regard to the procedure in ref. 12 refers to the surfactant removal. Now, it has been carried out by chemical extraction in order to favour lesser condensation of the nanoparticles, this leading to smaller aggregates that, in addition, can be more easily separated. Indeed, $1 \mathrm{~g}$ of mesostructured UVM-7 was suspended in a solution containing $90 \mathrm{~mL}$ of ethanol and $10 \mathrm{~mL}$ of $\mathrm{HCl}$, and this mixture was heated at $60{ }^{\circ} \mathrm{C}$ for $16 \mathrm{~h}$ while stirring. The resulting powder was collected by filtration, washed with water and ethanol, and air-dried.

The as-synthesized solid consist of UVM-7 micrometric aggregates. These were transformed into small submicrometric or nanometric aggregates by means of high power ultrasound treatment (using a Branson instrument). In a typical preparation, a suspension containing $1 \mathrm{~g}$ of UVM-7 in $100 \mathrm{~mL}$ of distilled water was irradiated for $15 \mathrm{~min}$ at a nominal power of $350 \mathrm{~W}$. After irradiation, the suspension has a colloidal character showing the Tyndall effect. The TEM images in Fig. 1 clearly show the significant decrease of the size of the aggregates after ultrasound treatment. To characterize these small UVM-7 particles, they were separated from the solution by ultracentrifugation. The fact that the solid has not been altered by the ultrasound treatment (i.e. it maintains the typical features of the UVM-7 mesoporous material) is confirmed by XRD, TEM and porosimetry $\left(S_{\mathrm{BET}}=1000 \mathrm{~m}^{2} \mathrm{~g}^{-1}\right.$ and two pore systems: small and large mesopores of 3 and $25 \mathrm{~nm}$, respectively). However, the soft chemical procedure used to remove the surfactant (in contrast to calcination in ref. 12) favours a low condensation degree of the silica particles, which is not significantly altered by the ultrasound irradiation according to the ${ }^{29} \mathrm{Si} \mathrm{NMR} \mathrm{spectra}$ $\left(\mathrm{Q}^{3} / \mathrm{Q}^{4}\right.$ ratio $\left.~ 1\right)$. This fact is exploited in the subsequent step to increase the nanoparticle connectivity on the polyurethane surface.

The foam mineralization is performed by successive impregnation cycles in the UVM-7 colloidal suspension followed by soft thermal treatment $\left(120^{\circ} \mathrm{C}\right.$ for $\left.2 \mathrm{~h}\right)$ to favour water evolution 


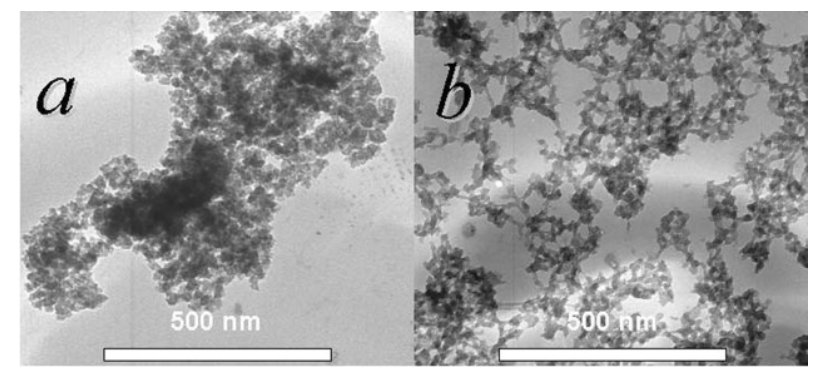

Fig. 1 TEM images of the UVM-7 aggregates before (a) and after (b) ultrasound treatment.

and nanoparticle condensation. To prepare the final trimodal porous material, the UVM-7/polyurethane composite was calcined at $500{ }^{\circ} \mathrm{C}$ for $5 \mathrm{~h}$. The macrocellular silica wall thickness readily increases with the number of impregnation treatments; after the third cycle, the calcined monoliths acquire a good rigidity and maintain the original shape of the foam.

The resulting porous monoliths present highly ramified networks defining interconnected large macropores with diameters ranging from hundreds of microns to $\mathrm{mm}$ (Fig. 2). The size of the macropores can be easily tailored at two different levels. A first modulation can be made, at a large scale, by using commercial polyurethane foams of different densities, and a second fine-tuning of the macropore sizes can be achieved by modifying the number of impregnation treatments. By increasing the number of cycles, the mineralization advances (as the coating of the foam), which leads to thicker walls and smaller macropores. Inspection of small pieces obtained by breaking the monoliths show that the regular macropore system is maintained in the whole body of the large monoliths. This fact indicates that the described procedure results in a homogeneous and regular coating of the organic foam. Each coating cycle gives rise to the deposit of regular UVM-7 films of about $5 \mu \mathrm{m}$. The foam used as template is commercial, inexpensive and allows a high dimensional control of the monolith size and shape.

High magnification SEM images (FE microscope) of the macroporous inorganic walls additionally reveal that the material surface consists of aggregates of soldered small primary nanoparticles, this generating a non-ordered system of large mesopores (Fig. 3a). On the other hand, the system of small mesopores is clearly observable in the TEM image in Fig. 3 b. The primary nanoparticles present an intra-nanoparticle hexagonal disordered mesopore system, characteristic of MCM-41-like solids, which is generated in the course of the cooperative self-assembling processes affecting the silicon precursor species and the surfactant micelles. Both large and small mesopore systems are typical of the UVM-7 silica used as reagent to construct the final trimodal monolith. Thus, everything indicates that, like after ultrasound nanodispersion, the subsequent interparticle condensation (impregnation plus thermal treatments) does not alter the typical UVM-7 architecture. Consistently with TEM observations, all monoliths display

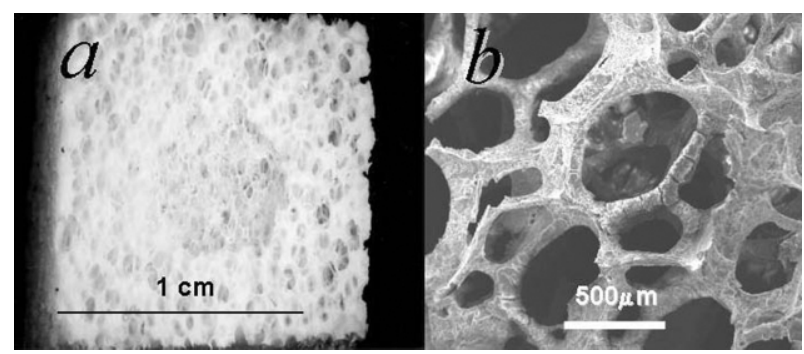

Fig. 2 (a) Optical photograph and (b) SEM image showing the monolithic nature and the macroporous system of the title materials.
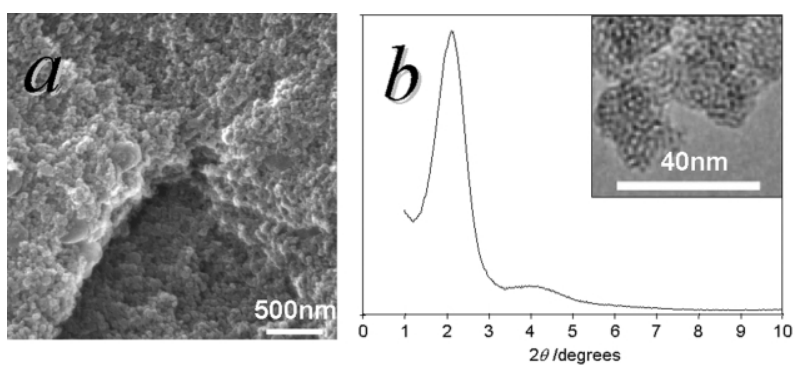

Fig. 3 (a) High resolution SEM photograph. (b) XRD pattern and TEM image showing the large and small mesopore systems.

XRD patterns with one strong peak and one broad signal of relatively low intensity, which is characteristic of disordered hexagonal pore arrays (Fig. 3b). Due to the large dimensions of the motive and the non-ordered nature of the large meso and macropore systems, the XRD data only inform about the intrananoparticle mesopores. The existence of a trimodal pore system with very high pore volume $\left(1.4 \mathrm{~cm}^{3} \mathrm{~g}^{-1}\right)$ in the UVM-7 foams leads to a site accessibility. The $\mathrm{N}_{2}$ adsorptiondesorption isotherms show two well-defined steps at intermediate and high $P / P_{0}$ values that are due to the condensation of nitrogen inside the intra-nanoparticle small mesopores and the interparticle large mesopores, respectively. $\dagger$

In conclusion, we report here on a simple preparative procedure yielding trimodal porous silica large monoliths having foam-like morphology. This approach has been extended to the preparation of similar silica-based monoliths admitting variable contents of different hetero-elements or organic groups starting from M-UVM-712 and HPNO organosilicas, ${ }^{13}$ respectively. The hierarchical pore system of these materials results in an enhanced accessibility to their active sites. Moreover, the versatility of the synthesis here presented, which is based on the successful isolation of mesoporous nanoparticles, allows its application to the preparation of large rigid monoliths by using scaffolds having good mechanic properties such as ceramic foams or vycor. ${ }^{14}$

This research was supported by the Spanish MCyT (MAT2000-1387-C02-01 and MAT2002-04239-C03-02).

\section{Notes and references}

1 M. Davis, Nature, 2002, 417, 813.

2 G. J. de A. A. Sler-Illia, C. Sanchez, B. Lebeau and J. Patarin, Chem Rev., 2002, 102, 4093 and references therein.

3 D. Zhao, P. Yang, B. F. Chmelka and G. D. Stucky, Chem. Mater., 1999 11, 1174 .

4 B. Zhang, S. A. Davis and S. Mann, Chem. Mater., 2002, 14, 1369 and references therein.

5 K. H. Rhodes, S. A. Davis, F. Caruso, B. Zhang and S. Mann, Chem. Mater., 2000, 12, 2832

6 B. T. Holland, L. Abrams and A. Stein, J. Am. Chem. Soc., 1999, 121 4308.

7 S. A. Bagshaw, Chem. Commun., 1999, 767.

8 Y.-J. Lee, J. S. Lee, Y. S. Park and K. B. Yoon, Adv. Mater., 2001, 13 95 and references therein.

9 S. A. Davis, M. Breulmann, K. H. Rhodes, B. Zhang and S. Mann, Chem. Mater., 2001, 13, 3218.

10 D. T. On and S. Kaliaguine, J. Am. Chem. Soc., 2002, 125, 618

11 P. Colombo, M. Griffoni and M. Modesti, J of Sol-Gel Sci. Tech., 1998, 13, 195.

12 J. El Haskouri, D. Ortiz de Zárate, C. Guillem, J. Latorre, M. Caldés, A. Beltrán, D. Beltrán, A. B. Descalzo, G. Rodriguez, R. Martínez-Mañez M. D. Marcos and P. Amorós, Chem. Commun., 2002, 330

13 J. El Haskouri, D. Ortiz de Zárate, C. Guillem, A. Beltrán, M. Caldes, M. D. Marcos, D. Beltrán, J. Latorre and P. Amorós, Chem. Mater., 2002 14, 4502

14 P. Amorós, A. Beltrán, D. Beltrán, C. Guillem, J. Latorre and L. Huerta, Spanish Patent 20030264. 